\title{
Processing Cycle Prediction Using Support Vector Regression in Intelligent Manufacturing
}

\author{
Wencan Tong, ${ }^{*}$ Hsien-Wei Tseng, and Zhiqiang Huang \\ School of Mathematics and Information Engineering, Longyan University, Fujian, 364012, China
}

(Received July 20, 2020; accepted January 6, 2021)

Keywords: big data technology, intelligent manufacturing equipment, processing cycle, cycle prediction

The processing cycle in an intelligent manufacturing machine (IMM) is difficult to predict accurately owing to uncertainties caused by unexpected maintenance errors and damage. Thus, a new method for accurate prediction is required. We propose a new prediction method using an algorithm based on support vector regression (SVR) in this study. The new method uses big data and determines its logical relationship with a processing cycle to obtain an accurate prediction of the cycle. The accuracy of the SVR method (>95\%) is better than that of the traditional method (79.3-89.6\%). The result proves that the method predicts the processing cycle accurately and provides essential information for developing algorithms for designing processing cycles in an IMM.

\section{Introduction}

The continuous development of big data technology is encouraging more and wider use of smart devices than before. Intelligent manufacturing (IM) has also been adopting big data technology in recent years for conveniently increasing productivity in the manufacturing industry. IM automatizes the design and manufacturing process of manufacturing machines via human-computer interaction. Various types of smart sensors are used for detectors, cameras, robotic arms, and so on, and provide collective data for the automation of IM. The data from machines are used for processing cycle prediction in IM.

In the design of an IM machine (IMM), processing cycle design has attracted the attention of many manufacturers. Accurate prediction of a processing cycle allows a better understanding of the production process, improves customer relations, and enables adaption to the dynamic situation of the market for sustainable growth. A processing cycle requires the compatibility of the components in an IM system to reduce the uncertainties originating from the complexity of the system. This makes accurate prediction difficult for a processing cycle of IMMs. ${ }^{(1)}$

In recent years, many researchers have focused on predicting processing cycles. Various methods for predicting processing cycles were proposed but they had accuracy problems that were a hurdle for the further development of IMMs. Therefore, we aim to propose a method of predicting a processing cycle using a support vector regression (SVR) method and validate the

*Corresponding author: e-mail: 853647745@qq.com

https://doi.org/10.18494/SAM.2021.3042 
result through an example analysis. The SVR method is expected to predict the processing cycle of an IMM effectively.

\section{Prediction Method of Processing Cycle}

\subsection{Logic of processing cycle}

A processing cycle of IM is established from the dependent and mutual relationship of processing activities based on the logic of the process. ${ }^{(2)}$ To improve the prediction accuracy of the processing cycle, the logical relationship is categorized into four different relations as shown in Fig. 1.

A serial relationship is the simplest logical relationship among the four relationships and has sequential processing steps. The completion of the previous processing steps is necessary for the next processing step. To predict the processing cycle, the original data is selected for an iterative calculation, ${ }^{(3)}$ and then the predicted data is added to the data of SVR as a logical relationship for short-term prediction. In a parallel relationship, several processes start at the same time. Only after the completion of the prior processes is the next process initiated. Therefore, the errors of multiple processes need to be corrected for the accurate prediction logic based on the established logical relationship. In a convergence relationship, the next processing cycle is predicted after the predictions of previous processing cycles are completed. A convergence relationship is the most common in predicting the processing cycle of an IMM. In a divergence relationship, a previously completed processing cycle is followed by multiple processing cycles.

The above four logical relationships are calculated using the random residual method (RRM). The effective logic of the RRM is expressed as

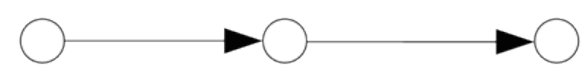

(a) Serial relationship

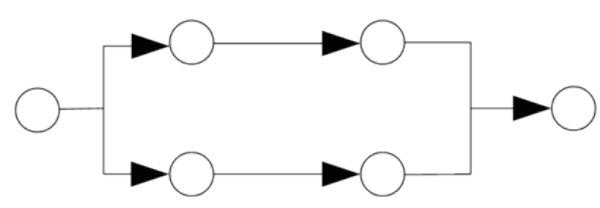

(b) Parallel relationship

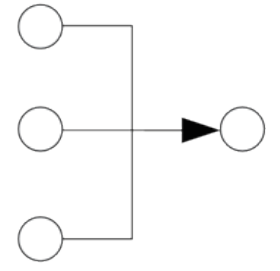

(c) Convergence relationship



(d) Divergence relationship

Fig. 1. Four logical relationships of the processing cycle of IM. 


$$
\gamma=\sum_{i=1}^{p}[f+h+k+l] / H_{p}
$$

where $\gamma$ represents the prediction logic in a processing cycle, $H_{p}$ represents the random residual coefficient, $f$ is a serial relationship, $h$ is a parallel relationship, $k$ is a convergence relationship, and $l$ is a divergence relationship. Equation (1) describes the logic for the accurate prediction of a processing cycle.

\subsection{SVR algorithm}

Big data may suffer from noise that can be removed by an SVR algorithm. An SVR algorithm is applied when there is a large divide between the upstream and downstream of a process due to a bottleneck and a QTime (clock time function) constraint. In this case, the algorithm improves the correlation between the cycle data and reduces the interaction between the predicted data. ${ }^{(4)}$ An SVR algorithm has high operating efficiency and tolerance for faults and noise with no influence on the interaction. ${ }^{(5)}$ A flow chart of the processing cycle prediction using an SVR algorithm is shown in Fig. 2.

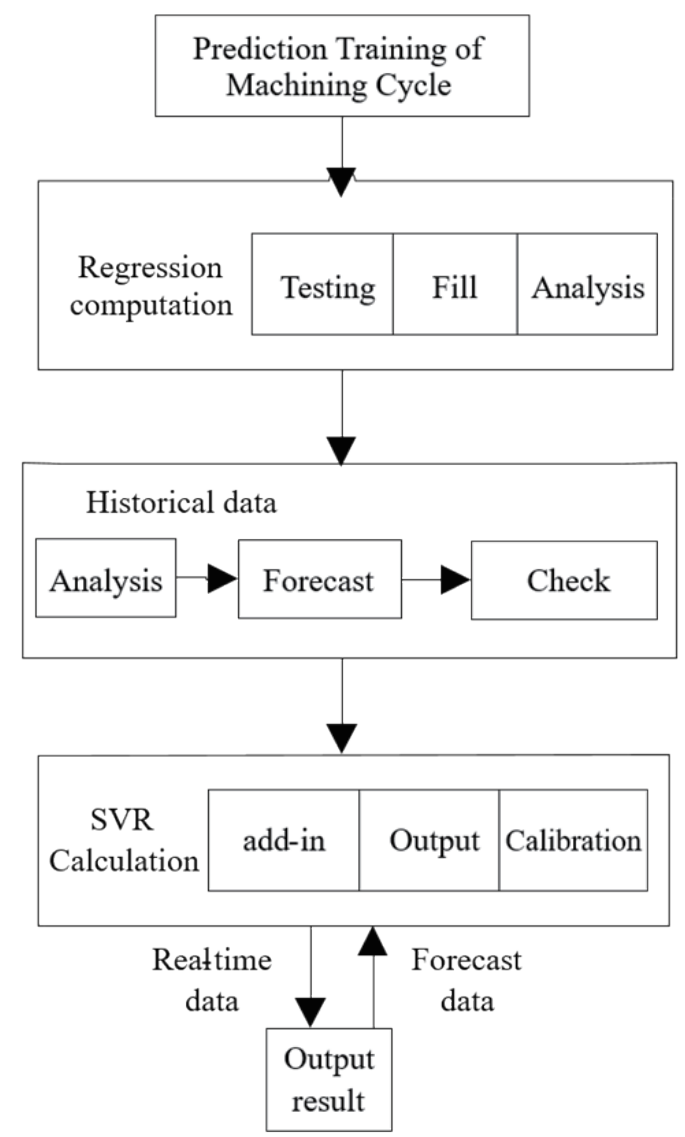

Fig. 2. Flow chart of predicting a processing cycle using an SVR algorithm. 
In Fig. 2, there are no sample parameters at the same level in the dimension reduction. The repeated prediction is based on the dimension reduction by the SVR algorithm, which improves the multicategory prediction of a processing cycle.

The SVR algorithm effectively enhances the accuracy of process cycle prediction by assigning different weights to the features, such as the duration of a process, frequency of use, and errors, and removing the features below thresholds. Thus, significant features are included in the process cycle prediction.

The essential idea of the SVR algorithm is to select the prediction sample $\gamma_{i}$ randomly from the periodic prediction logic $\gamma$, and then find $n$ prediction samples from $\gamma_{i}$. Then, the weight of each feature is updated according to the following rule to obtain a new weight for each feature.

$$
w(j)=\sum_{n} \frac{N M / n}{1-\left(\gamma_{i}\right)^{2}},
$$

where $w(j)$ is the weight of a predicted sample $\gamma_{i}$ for feature $j, N$ is the distance function, and $M$ is the number of times the predicted samples are randomly extracted.

By using $w(j)$, the SVR algorithm performs a sensitive prediction to ensure efficiency and accuracy. This is one of the advantages of the SVR algorithm in predicting nonlinear processing with small sample data. ${ }^{(6)}$ At the same time, the prediction calculation is completed together with the prediction logic and machine learning.

\subsection{Processing cycle prediction in IM}

Before a processing cycle is predicted, the components of the cycle must be analyzed. First, an instrumental processing cycle is required for estimating the time for accident inspection, decomposition, repair, and so on. The uncertainty in a processing cycle makes it difficult to predict the cycle accurately. ${ }^{(7)}$ Therefore, a processing cycle needs a distribution law and completion probability for its prediction. Second, a critical probability is required. This is the probability that a component exceeds a threshold (a critical line) during a process, ${ }^{(8)}$ and is a reference coefficient of the feature weight.

The key lines of the network are represented by $j_{1}, j_{2}, j_{3}, \ldots, j_{n}$. If the frequency in the singular line with a value of $n$ is $m_{1}, m_{2}, m_{3}, \ldots, m_{n}$, the processing probability is defined as

$$
\omega=w(j) / D, 1 \leq m_{n} \leq j_{n} \leq r,
$$

where $\omega$ represents the processing probability of the IMM, $D$ represents the processing key degree, $r$ represents the number of processing cycles in the prediction, and $m_{1}, m_{2}, m_{3}, \ldots, m_{n}$ and $j_{1}, j_{2}, j_{3}, \ldots, j_{n}$ are the reference coefficients of the current calculation.

The probability of the key line using the above equations is used to reduce the uncertainty of the processing cycle ${ }^{(9)}$ and determine the probability distribution and the parameters of each process. The prediction model of a processing cycle includes the probability distribution of each process. The equation for the model is as follows: 


$$
F(\mu)=\sum_{i=1}^{n} \gamma_{i} \omega p_{i}
$$

where $F$ is the function of the processing cycle prediction model, $p_{i}$ is the number of the instrument in the processing cycle, and $\mu$ represents the direct output value.

An SVR algorithm using this model is then employed to predict the processing cycle. The iterative calculation is repeated until the error reaches zero, and then the frequency distribution and processing cycle are obtained.

\section{Results and Discussion}

To verify the validity and feasibility of processing cycle prediction using the SVR algorithm, an analysis was carried out using the new method and the traditional prediction method.

\subsection{Application process}

Two methods were applied to the man-hour distribution of a processing cycle to determine the accuracy of the prediction. The data was collected from a smart factory of construction machinery. First, a network plan for the processing cycle was established by using Monte Ball software. ${ }^{(10)}$ The distribution of man-hours in the processing cycle was different for the different processes of the cycle (Table 1). In the repeated processing cycle, each process has three different man-hours. ${ }^{(11)}$

The processing time (man-hours) in Table 1 shows that each process has a single constraint relation from which the logical relationship of the instrumental processing cycle is established. The distance function $N=0.95$ and the logical relationship $\gamma$ are calculated by the RRM. The logical relationship allows the calculation of the accuracy for the processing cycle prediction. ${ }^{(12)}$

Table 1

Man-hour distribution of processing cycle.

\begin{tabular}{lccc}
\hline Process code & Process & Prior process & Man-hour distribution \\
\hline A & Check spare pump & A & $(28,32,59)$ \\
B & Calibrate all pipes & A & $(84,26,54)$ \\
C & Remove pump cover and rotor & B & $(25,69,63)$ \\
D & Clean pipes and lines & E & $(31,54,98)$ \\
E & Replace piping & $\mathrm{C}$ & $(16,94,35)$ \\
F & Recover impeller performance & $\mathrm{C}$ & $(20,54,94)$ \\
G & Repair pump bearings & $\mathrm{C}$ & $(3,26,48)$ \\
H & Balance impeller & $\mathrm{H}$ & $(58,4,26)$ \\
$\mathrm{I}$ & Check swirl bearing & $\mathrm{I}, \mathrm{J}, \mathrm{K}$ & $(9,29,46)$ \\
$\mathrm{J}$ & Repair vortex rotor & $\mathrm{D}$ & $(31,1,44)$ \\
$\mathrm{K}$ & Test parts and components & $\mathrm{D}$ & $(16,0,89)$ \\
$\mathrm{L}$ & Install seal packing & $\mathrm{D}$ & $(41,63,61)$ \\
$\mathrm{M}$ & Attempt operation & $\mathrm{L}$ & $(20,58,71)$ \\
\hline
\end{tabular}


Table 2

Comparison of processing cycle prediction results.

\begin{tabular}{|c|c|c|c|c|c|}
\hline \multirow{2}{*}{$\begin{array}{l}\text { Completion } \\
\text { probability }\end{array}$} & \multirow{2}{*}{$\begin{array}{c}\text { Actual processing } \\
\text { hours }(\mathrm{h})\end{array}$} & \multicolumn{2}{|c|}{ SVR method } & \multicolumn{2}{|c|}{ Traditional method } \\
\hline & & Predicted hours (h) & Accuracy $(\%)$ & Predicted hours (h) & Accuracy $(\%)$ \\
\hline 0.1 & 461 & 479 & 96.2 & 514 & 89.6 \\
\hline 0.2 & 502 & 516 & 97.3 & 398 & 79.3 \\
\hline 0.3 & 210 & 214 & 98.1 & 168 & 80 \\
\hline 0.4 & 340 & 341 & 99.7 & 301 & 88.5 \\
\hline 0.5 & 402 & 412 & 97.5 & 354 & 88.1 \\
\hline 0.6 & 395 & 401 & 98.5 & 484 & 81.6 \\
\hline 0.7 & 485 & 490 & 98.9 & 415 & 85.4 \\
\hline 0.8 & 365 & 350 & 95.8 & 291 & 79.6 \\
\hline 0.9 & 535 & 536 & 99.8 & 442 & 82.6 \\
\hline 1.0 & 314 & 315 & 99.6 & 359 & 87.4 \\
\hline
\end{tabular}

\subsection{Analysis of results}

Under the same conditions, the prediction by simulating multiple machine cycle operations was performed on the IMM in the smart factory. Table 2 shows ten predictions of the processing cycle and the times predicted by the SVR and the traditional method at each level of completion probability.

The analysis results show that the prediction accuracy of the SVR method is consistently higher than that of the traditional method with an average difference of $15.7 \%$. The difference between the predicted and actual hours in the SVR method does not exceed $20 \mathrm{~h}$, which is much smaller than that in the traditional method $(>39 \mathrm{~h}$ ). In the actual processing cycle, the prediction using the traditional method has limitations due to uncertainties from varying processing time, imprecise engineering control, damage to machines, and the complicated logical relationship in the process. The prediction of the processing cycle by the SVR method has an accuracy of $95.8-99.8 \%$, while that by the traditional method is less than $89.6 \%$. The results indicate that the prediction of the processing cycle requires information on processing conditions and procedures for a more accurate result.

\section{Conclusion}

A new method of processing cycle prediction using an SVR algorithm was proposed in this study. This method enables accurate prediction of the processing cycle in an IMM. The accuracy at each completion probability was higher than $95 \%$ for the SVR method, while it was $79.3-89.6 \%$ for the traditional method. The relative error of the proposed prediction method was $7.5 \%$, which was much less than that of the traditional method (65.7\%). Despite the fact that many uncertainties complicate a manufacturing process and the logical relationship in the process, the SVR method showed a high prediction accuracy. The proposed method is expected to lead to more research to provide a theoretical basis and reference for the prediction of periodic processing cycles. 


\section{Acknowledgments}

We are grateful for financial support from the Youth Climbing Project of the Longyan University (LQ2016004) and the Youth Project of Fujian Province Education Hall (JT180522).

\section{References}

1 C. C. Wang, C. X. Wang, and Z. Liu: China Standardization 15 (2018) 101 (in Chinese). https://kns.cnki.net/ $\mathrm{kcms} /$ detail $/$ detail. asp $x$ ?dbcode $=$ CJFD $\&$ dbname $=$ CJFDLAST2018\&filename $=$ ZGBZ201804004\&v $=\% 25 \mathrm{mmd}$ 2BcETbXm\%25mmd2F2oEgPapPN1D9LskCZlp0YzGPMCJoARTvnOuFk59eFAlXCRy84B8VhQRa

2 F. Xu: Autom. Expo. 26 (2016) 72 (in Chinese). https://kns.cnki.net $/ \mathrm{kcms} / \mathrm{detail} / \mathrm{detail} . \mathrm{aspx}$ ?dbcode=CJFD\&d bname $=$ CJFDLAST2016\&filename $=$ ZDBN201601036\&v $=1 \mathrm{pT} 31$ OYsFn4oNjlx4wui\%25mmd2FJCnzZf7oA8lb PSigcL\%25mmd2ByUyVmGo8u\%25mmd2FJJ0hvrypKqEdzk

3 Y. Lu and J. D. Yin: Mach. Tools Hydraul. Pressure 46 (2018) 111. http://en.cnki.com.cn/Article_en/CJFDTotalJCYY201801001.htm

4 J. R. Gao, Q. Gao, and F. Gao: Intell. Manuf. 32 (2018) 159 (in Chinese). https://kns.cnki.net/kcms/detail/ detail.aspx .dbcode $=$ CJFD $\&$ dbname $=$ CJFDLAST2018\&filename $=J$ SFY201801043\&v $=$ ETerGyrgb $\% 25 \mathrm{mmd} 2 \mathrm{~F}$ NI7tGeJKvHfZz\%25mmd2FGDQf6y 5 LWopy $\% 25 \mathrm{mmd} 2 \mathrm{Bi} \% 25 \mathrm{mmd} 2 \mathrm{BAUyocyjxNDoxdpco250q001Xd}$

5 M. L. Zhang: Ship Sci. Technol. 38 (2016) 121 (in Chinese). https://kns.cnki.net/kcms/detail/detail.aspx?dbcod $\mathrm{e}=$ CJFD\&dbname $=$ CJFDLAST2016\&filename $=J C K X 201609027 \& v=B J 1 \mathrm{kxnbYMYhFbPC4vf7I2ENujr3E} \% 2$ 5mmd2BQDEiebT2tooesxUlvstMbAoJv29E8EX9T8f

6 Z. Chen and X. L. Jia: Comput. Integr. Manuf. Syst. 24 (2018) 132 (in Chinese). https://kns.cnki.net/kcms/ detail/detail.aspx?dbcode $=$ CJFD\&dbname $=$ CJFDLAST2018\&filename $=J S J J 201802001 \& v=e c B Z s C d v Y Y T 8 V$ gtDaENVhL7BDY2Ycl20qQmolTMxxmBZTGUeiJUcW\%25mmd2F8F5jTHamkG

7 X. C. Zhu and F. Qiao: Comput. Integr. Manuf. Syst. 23 (2017) 2172. https://xueshu.baidu.com/usercenter/ paper/show?paperid=a710f6b5d3d8d3670741e9ef713c140e\&site=xueshu se

8 D. H. Zou, S. C. Chai, and B. W. Wang: J. Yangtze Univ. (self-edition) $\mathbf{1 2}$ (2018) 82 (in Chinese). https://kns. cnki.net $/ \mathrm{kcms} /$ detail $/$ detail.aspx?dbcode $=$ CJFD $\&$ dbname $=$ CJFDLAST2018\&filename $=$ CJDL201803015\&v $=$ GGiK9sejTBzmvVTD0etmZ\%25mmd2BsVdtrjwR34Qs7QHIUluQzQH7D\%25mmd2FutckQyN9rRgDjphk

9 L. Lin, X. Z. Chen, and S. S. Zhong: J. Harbin Univ. Technol. 48 (2016) 27. https://kns.cnki.net/KNS8/Detail?s field=fn\&QueryID $=75 \&$ CurRec $=1 \&$ recid $=\&$ FileName $=$ HEBX201607004\&DbName $=$ CJFDLAST2016\&DbCo $\mathrm{de}=\mathrm{CJFD} \& \mathrm{yx}=\mathrm{Y} \& \mathrm{pr}=\& U R L I D=23.1235 . \mathrm{t} .20160608 .1623 .048$

10 F. Zhou and C. Peter: Comput. Sci. 43 (2016) 119. https://xueshu.baidu.com/usercenter/paper/show?paperid=56 f7e8df7a82b448c9036cdcacf5b3fb\&site=xueshu se

11 J. Li and N. Chen: China Manage. Sci. 30 (2016) 790 (in Chinese). https://kns.cnki.net/kcms/detail/detail.aspx? $\mathrm{dbcode}=\mathrm{CJFD} \& \mathrm{dbname}=\mathrm{CJFDLAST} 2017 \&$ filename $=$ ZGGK2016S1111\&v $=\mathrm{JFQm} \% 25 \mathrm{mmd} 2 \mathrm{FyrXvvamw9ZU}$ FrzkdZxNe4GAm\%25mmd2B783hcZ90EOJE65QzfuXk45XjE0h9iD0wPQ

12 X. W. Ma, H. Xue, and J. M. Qi: J. Shenyang Architectural Univ. (Natural Science Edition) 72 (2018) 75 (in Chinese). https://kns.cnki.net $/ \mathrm{kcms} /$ detail/detail.aspx?dbcode $=$ CJFD\&dbname $=$ CJFDLAST2018\&filename $=\mathrm{S}$ YJZ201801004\&v=MirOhdiI4140Q4qDsuZ4ThxeYSUs0NzYPYJJdVcJypn7FS0vNFleWqW251AeKO4n 